\title{
Impact of the SARS-CoV-2 pandemic on turnover time and revenue in the endoscopy unit: single-center experience ${ }^{*}$
}

\section{다 (1) $\odot$}

\author{
Authors \\ Abdelhai Abdelqader, Avik Sarkar, Haroon Shahid, Amy Tyberg, Sohini Sameera, Mihajlo Gjeorgjievski, Karoline \\ Escobedo, Alexa Simon, Romy Bareket, Ping He, Eric Zhao, Monica Gaidhane, Michel Kahaleh
}

Institution

Department of Medicine, Division of Gastroenterology, Robert Wood Johnson Medical Center, New Brunswick, New Jersey, United States

submitted 12.3.2021

accepted after revision $\quad 17.6 .2021$

\section{Bibliography}

Endosc Int Open 2021; 09: E1680-E1685

DOI 10.1055/a-1546-8302

ISSN 2364-3722

(c) 2021. The Author(s).

This is an open access article published by Thieme under the terms of the Creative Commons Attribution-NonDerivative-NonCommercial License, permitting copying and reproduction so long as the original work is given appropriate credit. Contents may not be used for commercial purposes, or adapted, remixed, transformed or built upon. (https://creativecommons.org/licenses/by-nc-nd/4.0/)

Georg Thieme Verlag KG, Rüdigerstraße 14,

70469 Stuttgart, Germany

\section{Corresponding author}

Michel Kahaleh, MD, Professor of Medicine, Clinical Director of Gastroenterology, Robert Wood Johnson University Hospital, New Brunswick, NJ 08901, United States

Fax: +1-732-235-7307

mkahaleh@gmail.com

\section{ABSTRACT}

Background and study aims The SARS-CoV-2 pandemic heavily impacted the New York metro area causing most in- stitutions to either reduce case volume or fully close remaining open units incorporated specific guidelines for procedures lockdown potentially leading to a greater turnover time. We analyzed the quantitative and financial impact of this lost time on our tertiary care center's endoscopy unit.

Patients and methods This single-center, retrospective study included demographics, procedure details and turnover times (TOT) from all endoscopic procedures between December 1, 2019 to June 30, 2020. Cases were categorized as pre-COVID-19 group from December 1, 2019 to March 15, 2020 and during COVID-19 (lockdown) group from March 16, 2020 to June 30, 2020. The financial impact was assessed using national averages for reimbursement of outpatient endoscopic procedures provided by Center for Medicare and Medicaid Services.

Results A total of 3622 procedures were performed during the study period: 2297 in the pre-COVID-19 period, 1325 in the COVID-19 period, representing a $42.32 \%$ decrease. In the COVID-19 lockdown group, there was a significant increase in TOT in both the general endoscopy cases (18.11 minutes, $P=0.000)$ and advanced endoscopy cases (17.7 minutes, $P=0.000$ ). The $42.3 \%$ decrease in volume equated to at least \$1.6 million USD in lost revenue during the lockdown.

Conclusions COVID-19 pandemic led to an increase in TOT with overall reduced procedure volume and a negative effect on revenue. Providing continued endoscopic management during a pandemic avoids delays in reopening the endoscopy unit and hampers the post-pandemic surge of delayed cases and its financial impact.

\section{Introduction}

SARS-CoV-2 disease has strongly impacted the New York metropolitan area, with the peak of the pandemic in the region occurring between March and May 2020 [1]. Many regional private practices and institutions had either significantly reduced case volume or fully closed their endoscopy units. During the

\footnotetext{
* Meeting presentations: Digestive Disease Week, 2021
}

surge of the COVID-19 epidemic, people were strongly encouraged to avoid hospitals and only emergency procedures were performed.

Indeed, the virus is predominantly spread by respiratory droplets, but fecal excretion has been reported [2]; upper and lower endoscopies became a focus for infection control. Gastrointestinal societies and the World Health Organization (WHO) released statements to maintain safety for healthcare personnel and patients in endoscopy units [3-6]. These guidelines includ- 
ed the strong recommendation to only perform urgent or emergent cases, defined as one or more of the following: 1) upper and lower gastrointestinal bleeding leading to symptoms; 2) dysphagia impacting oral intake; 3) cholangitis or impending cholangitis; 4) symptomatic pancreaticobiliary disease, i.e. necrotizing pancreatitis; 5) palliation of gastrointestinal obstruction; 6) patients with a time-sensitive diagnosis, such as premalignant or malignancy; 7) cases in which endoscopic procedures would urgently change management such as in IBD; and 8) other cases reviewed and deemed to be exceptional $[4,6]$.

Hospital factors such as increased need for enhanced personal protective equipment, reduced staff and room capacity, need for protective pathways ensuring linear flow of patients through units, and enhanced cleaning coupled with environmental factors such as accessories and device delivery issues and COVID-19 testing caused a substantial reduction in services. As a result of these changes made during the pandemic, we hypothesized that the turnover time (TOT) between patients was also affected.

TOT, which was defined as the time the patient is transported out of the room to the time the next patient is transported into the room, is a mainstay focus in our study. The TOT is crucial to every endoscopy unit, as optimization of this time has shown to improve endoscopist performance measures as well as endoscopy efficiency [7]. The demand for endoscopy services is high; improving general TOT may be an important potential mechanism to meet this high demand. Improving an endoscopy unit's efficiency could have major benefits in its cost effectiveness. As we learned more about COVID-19, endoscopy units started reopening or increasing their capacity; however, many of the changes implemented during the pandemic remain in effect in endoscopy units.

We aimed to measure the impact of COVID-19 on the endoscopy unit at our tertiary care center in the New York metropolitan area (New Brunswick, New Jersey, United States) and the financial implications related to it.

\section{Patients and methods}

Data were retrieved from our endoscopy quality activities (QA) QC) database. The database was established to assess and improve the quality of the program to ensure established educational, clinical service standards are met or best evidentiary practices attained. We reviewed inpatient and/or outpatient endoscopic procedures during the lockdown period of March 16, 2020 to June 30, 2020. Patients who underwent inpatient and/or outpatient endoscopic procedures during the proceeding months were used as a comparative baseline, from December 1, 2019 to March 15, 2020. Demographics, quantity of procedures, procedure types, and TOT were obtained.

TOT was defined as the time the patient is transported out of the room to the time the next patient is transported into the room. This did not include time needed to intubate the patient nor the procedure time. A two-sample $t$-test was conducted to compare TOT. Interventional endoscopic cases were reviewed as a subset, which included but were not limited to mucosal and submucosal dissection, transoral fundoplication, and endoscopic ultrasound. The financial impact on endoscopic procedures was assessed using national averages for reimbursement for outpatient endoscopic procedures provided by Center for Medicare and Medicaid Services (CMS) [8-12]. In regards to analyzing the financial impact, our data included outpatient and inpatient procedures.

During the lockdown period, only emergent/urgent procedures were performed. There were two socially distanced preoperative and postoperative bays and up to two endoscopy rooms utilized. All endotracheal intubations were done with a Hepa filter in place to limit the spread of the virus in the procedure area. There were at least two endoscopists (one interventionalist) always present. There was no more than one fellow per case. Nursing staff was reduced to no more than two per case. Total nurses and technicians per day was about 13 . The number of anesthesiologists remained unchanged. There was no shortage of personal protective equipment (PPE) such as N95 masks or powered air purifying respirators (PAPRs). After each procedure, an extensive room-cleaning protocol was implemented and all horizontal and high-touch surfaces, such as procedure tables, countertops, chairs, patient care equipment, and anesthesia equipment, were thoroughly wiped

\section{Results}

A total of 3622 procedures were performed during the study period: 2297 during the pre-COVID-19 period and 1325 during the COVID-19 lockdown, representing a $42.32 \%$ decrease. The quantity of both general and interventional endoscopic procedures decreased ( $\mathbf{F i g . 1 a , ~}$ - Fig. 1b). There were 1139 (49\%) and 735 (55\%) male patients in the pre-COVID-19 and COVID19 lockdown groups, respectively, with a mean age of 59 years. The mean TOT from pre-COVID-19 to during COVID-19 increased by $45 \%$ from 40.2 minutes to 58.3 minutes ( $\triangleright$ Table 1). In the COVID-19 lockdown group, there was a significant increase in TOT in both the general endoscopy cases $(18.11 \mathrm{~min}$ utes, $P=0.000)$ and advanced endoscopy cases (17.7 minutes, $P=0.000$ ) ( Table 1). Furthermore, during the COVID19 lockdown period, the time between a general case and an interventional case had a statistically significant increase (9.79 minutes, $P=0.012$ ) ( Table 1).

The decrease in nursing staff and extensive room cleaning process was responsible of the prolonged TOT. The number of cases performed per day steadily increased 2 months into the pandemic ( $\mathbf{F i g . 2}$ ). Of eight endoscopists, five became COVID-19 positive during the lockdown, but none required hospitalization.

The $42.3 \%$ decrease in volume equated to at least $\$ 1.6$ million USD in lost revenue during the lockdown. This represented $\$ 960,000$ USD from general endoscopy cases and $\$ 963,000$ USD from interventional cases ( $\triangleright$ Table 2 , $\triangleright$ Table 3 ).

\section{Discussion}

As healthcare systems became flooded with high volumes of patients with COVID-19 during the pandemic, medical centers had to further improve efficiency while maintaining high-qual- 

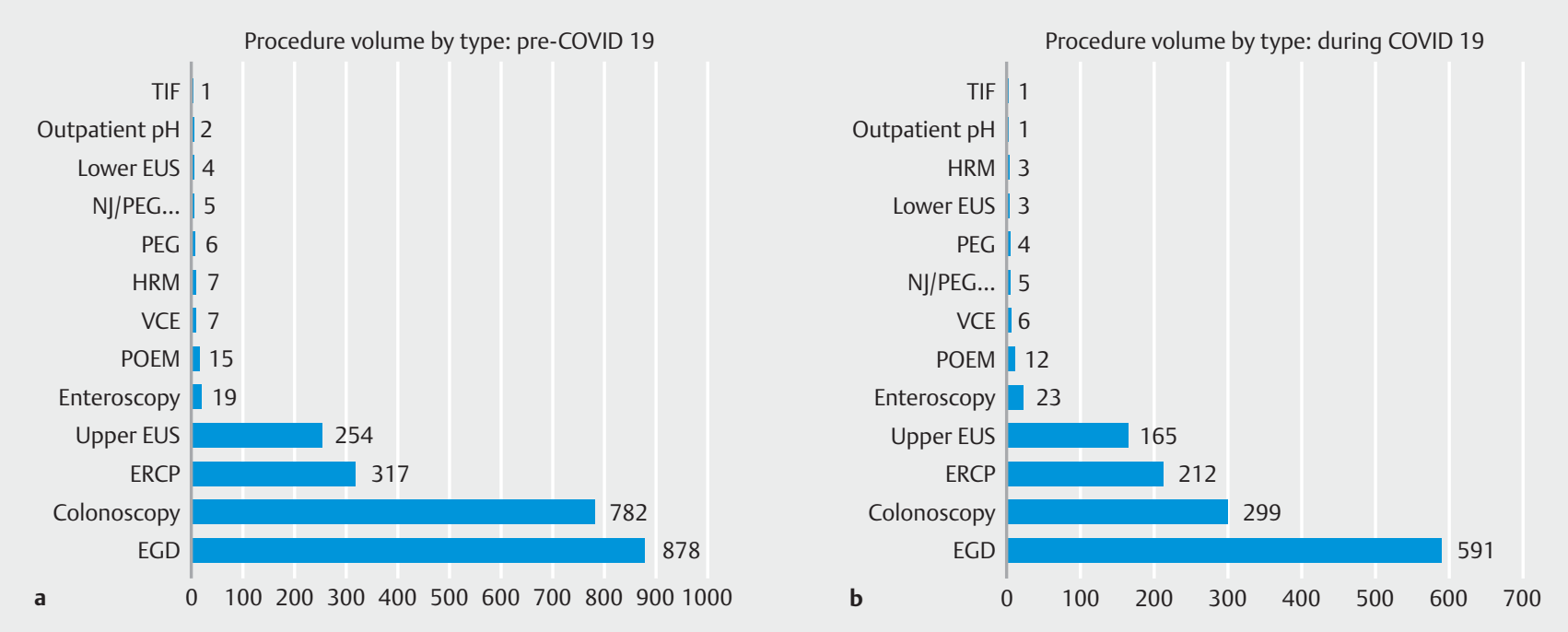

- Fig. 1 a Endoscopy procedure volume by type: period before COVID-19. b Endoscopy procedure volume by type: period during COVID-19.

- Table 1 Endoscopy unit patient demographics and case productivity.

\begin{tabular}{|l|l|l|}
\hline & Pre-COVID-19 & COVID-19 lockdown \\
\hline Average age (years) & 58 & 60 \\
\hline Sex (male) & $1139(49 \%)$ & $735(55 \%)$ \\
\hline Number of procedures (n) & 2297 & 1325 \\
\hline Number of advanced procedures (n) & 678 & 481 \\
\hline Average turnover times (minutes) & Average (SD) & Average (SD) \\
\hline - Composite & $40.2(23.5)$ & $58.3(33.4)$ \\
\hline - General & $36.7(23.2)$ & $54.8(33.4)$ \\
\hline - Advanced & $47(22.4)$ & $64.6(32.2)$ \\
\hline SD, standard deviation. & & \\
\hline
\end{tabular}

ity care. Many regional private practices and institutions had either significantly reduced case volume or fully closed their endoscopy units [13]. During the peak of the pandemic, our institution was one of the most heavily affected facilities in our region. Yet, our endoscopy unit remained functional throughout the pandemic, providing emergent patient care when medical access became restricted. However, the decrease in nursing staff and implementation of an extensive room cleaning protocol after each endoscopy dramatically affected TOT.

Our analysis revealed that TOT increased 1.5 times in the COVID-19 lockdown group, and case volume decreased by $42 \%$. Although this decrease reflected only cases deemed emergent or urgent, it was higher than we expected because neighboring facilities were shut down and referred their patients to our center because we are a tertiary referring center in the area. No infections occurred in our unit after hospitalwide infection control measures were put in place. In a recent study from Italy, the global number of procedures in tertiary centers decreased from 19,876 in March 2019 to 7701 (61.2\% decrease rate) in March 2020. The number of esophagogastro- duodenoscopies decreased from 8967 to 3143 (64.9\%) and rectosigmoidocolonoscopies decreased from 9563 to 3795 $(60.3 \%)$. The reduction was slightly less pronounced for pancreaticobiliary endoscopy procedures, with endoscopic ultrasound decreasing from 732 to 407 (44.4\%) and endoscopic retrograde cholangiopancreatography from 614 to 356 (42\%) [14], similar to our data.

In analyzing the financial impact of the lockdown period on our endoscopy unit, the preceding 3 months were used for comparison due to our center's steadily increasing volume. Outpatient CMS reimbursement estimates were used, but the authors acknowledge the amount of reimbursement varies per practice and each endoscopy can entail a wide array of endoscopic interventions. Financially, we estimated at least \$1.6 million USD in reduced total revenue for all endoscopic procedures compared to the preceding months.

Remaining open throughout the pandemic allowed our facility to reduce the financial impact compared to being completely shut down. 
- Table 2 Components of total procedures performed and average outpatient reimbursement by CMS (excluding interventions, including sphincterotomy and stent placement).

\begin{tabular}{|l|l|l|l|l|l|}
\hline Procedure type & Pre-COVID-19 & COVID-19 lockdown & Cost (USD) & Total (pre) & Total (lockdown) \\
\hline EGD & 878 & 591 & 762 & 669,036 & 450,342 \\
\hline Colonoscopy & 782 & 299 & 980 & 766,360 & 293,020 \\
\hline Upper EUS & 254 & 165 & 1483 & 376,682 & 244,695 \\
\hline Lower EUS & 4 & 3 & 980 & 3,920 & 2,940 \\
\hline ERCP & 317 & 212 & 2825 & 895,525 & 598,900 \\
\hline VCE & 7 & 6 & 786 & 5,502 & 4,716 \\
\hline Enteroscopy & 19 & 23 & 1483 & 28,177 & 34,109 \\
\hline PEG & 6 & 4 & 1483 & 8,898 & 5,932 \\
\hline Outpatient pH & 2 & 1 & 485 & 970 \\
\hline HRM & 7 & 3 & 485 & 3,395 & 1,455 \\
\hline POEM & 15 & 12 & 2500 & 37,500 & 30,000 \\
\hline NJ/PEG exchange & 5 & 5 & 231 & 1,155 & 1,155 \\
\hline TIF & 1 & 1 & 3613 & 3,613 & 3,613 \\
\hline Total & & & & $2,800,733$ \\
\hline CMS, Cen & & & & $1,671,362$ \\
\hline
\end{tabular}

CMS, Center for Medicare and Medicaid Services; EGD, esophagogastroduodenoscopy; EUS, endoscopic ultrasound; ERCP, endoscopic retrograde cholangiopancreatography; VCE, video capsule endoscopy; PEG, percutaneous endoscopic gastrostomy; HRM, high-resolution manometry; TIF, transoral incisionless fundoplication. POEM; per oral endoscopic myotomy.

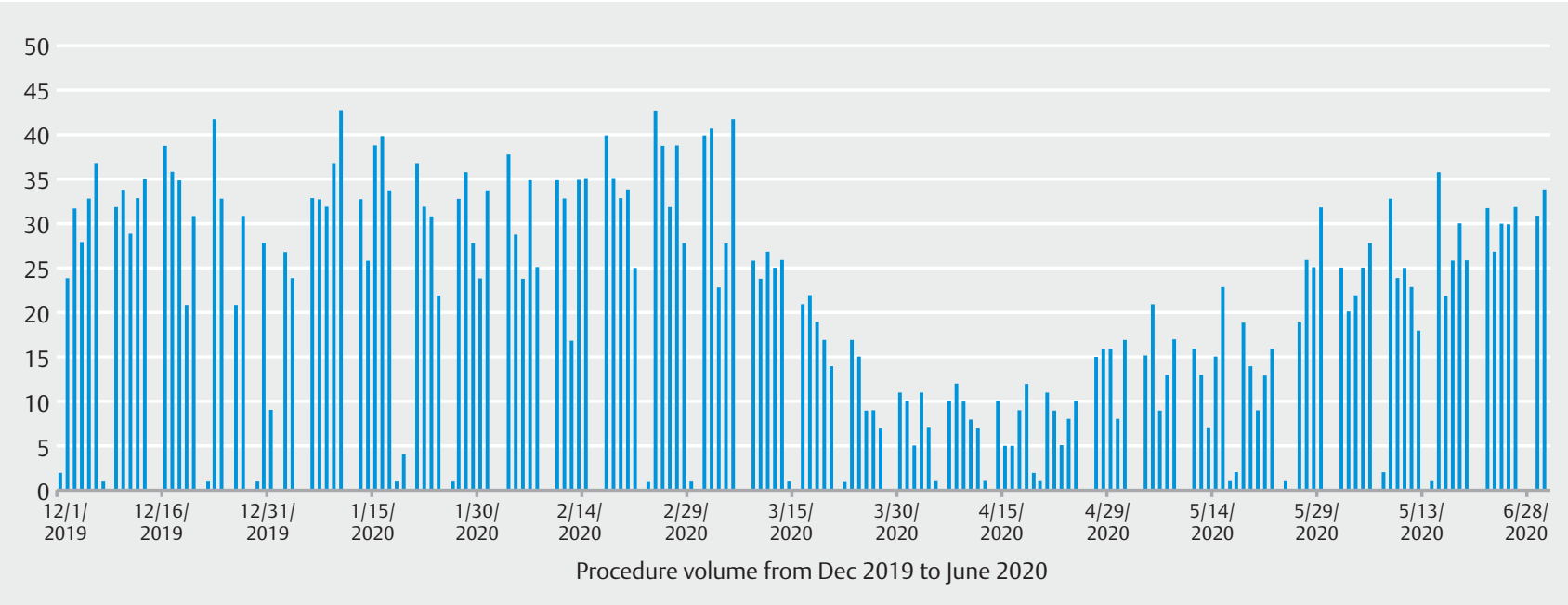

- Fig.2 Endoscopy procedure volume from December 2019 through June 2020.

We observed local facilities that shut down during the pandemic had delays reopening and met a lot of obstacles in revising their infection control processes. In addition, some healthcare providers chose not to return to work because of fear of getting infected. By adapting infection control measures based on the evolving infection control recommendations, our center avoided not only delays in reopening the endoscopy unit but the post-pandemic surge of delayed cases and its resultant financial impact. Due to the adaptive infection control measures, after the lockdown period, our endoscopy unit rapidly returned to being fully operational.

Our study had a few limitations. The optimal workflow processes could not be reported, as recommendations continued to be modified throughout the study period. The financial impact was significant but we could not account for all accessories and devices utilized, such as biliary stents, biopsy forceps, injection needles wires, and luminal stent deployment). Our facility fees may be different from those in other centers. However, we feel it is essential to report our experience as a heavily im- 
- Table 3 Components of all interventional endoscopic procedures and average outpatient reimbursement by CMS.

\begin{tabular}{|l|l|l|l|l|l|}
\hline Procedure type & Pre-lockdown & Lockdown & Cost & Total (pre-lockdown) & Total (lockdown) \\
\hline EGD & 46 & 45 & 762 & 35,052 & 34,290 \\
\hline Colon & 11 & 13 & 980 & 10,780 & 12,740 \\
\hline Upper EUS & 254 & 165 & 1483 & 376,682 & 244,695 \\
\hline Lower EUS & 4 & 3 & 980 & 3,920 & 2,940 \\
\hline ERCP & 317 & 212 & 2825 & 895,525 & 598,900 \\
\hline TIF & 1 & 1 & 3613 & 3,613 & 3,613 \\
\hline POEM & 15 & 12 & 2500 & 37,500 & 30,000 \\
\hline Enteroscopy & 19 & 23 & 1483 & 28,177 & 34,109 \\
\hline NJ/PEG Exchange & 5 & 5 & 231 & 1,155 & 1,155 \\
\hline Outpatient pH & 2 & 1 & 485 & 970 & 485 \\
\hline EndoFLIP & 4 & 1 & 485 & 1,940 & 485 \\
\hline
\end{tabular}

pacted center that remained open throughout the COVID-19 epidemic.

Finally, by adapting infection control measures based on continued infection control recommendations during the COVID-19 pandemic, our center prevented not only delays in reopening the endoscopy unit but the post pandemic surge in delayed cases and its resultant financial impact.

\section{Conclusions}

In conclusion, the COVID-19 pandemic led to an overall reduced procedure volume and an increase in TOT with a negative effect on revenue. As we enter the second year of the COVID-19 pandemic, these data underscore the need to maintain a functioning endoscopy unit while adopting infection control measures.

\section{Competing interests}

Dr. Tyberg is a consultant for Endogastric Solutions. Dr. Gaidhane is a consultant for Interscope Med. Dr. Kahaleh receives grant support from Boston Scientific, MaunaKea, Apollo Endosurgery, Cook Endoscopy, ASPIRE Bariatrics, NinePoint Medical, Merit Medical, Olympus, and Interscope Med and is a consultant for Boston Scientific, Concordia Laboratories Inc., Abbvie, and Mauna Kea Tech.

\section{References}

[1] Allen J, Almukhtar S, Aufrichtig A et al. New Jersey Coronavirus Map and Case Count. The New York Times; 19.05.2021 Available at: https://www.nytimes.com

[2] Cheung K, Hung I, Chan P et al. Gastrointestinal manifestations of SARS-CoV-2 infection and virus load in fecal samples from the Hong Kong cohort and systematic review and meta-analysis. Gastroenterology 2020; 159: 81-95

[3] Guidance for resuming Gl endoscopy and practice operations after the COVID-19 Pandemic [PDF file]. American Society for Gastrointes- tinal Endoscopy; 2020: Available at: https://www.asge.org/docs/default-source/default-document-library/asge-guidance-for-reopeningl_4-28-2020.pdf

[4] Gastroenterology professional society guidance on endoscopic procedures during the COVID-19 pandemic [PDF file]. American Society for Gastrointestinal Endoscopy; 2020: Available at: https://webfiles. gi.org/links/media/Joint_Gl_Society_Guidance_on_Endoscopic_Procedure_During_COVID19_FINAL_impending_3312020.pdf

[5] Pochapin M, Greenwald D. COVID-19 update \& recommendations for gastroenterologists. American College of Gastroenterology; Available at: https://mailchi.mp/gi/acgrecommendations-re-covid-19-for-gastroenterologists? $=$ dbeba47cbc

[6] Rational use of personal protective equipment for coronavirus disease (COVID-19): interim guidance [PDF file]. World Health Organization; 2020: Available at: https://www.who.int/publications/i/item/rational-use-of-personal-protective-equipment-for-coronavirus-disease(covid-19)-and-considerations-during-severe-shortages

[7] Berg B, Denton B, Nelson H et al. A discrete event simulation model to evaluate operational performance of a colonoscopy suite. Med Decision Making 2010; 30: 380-387

[8] 2020 MPFS proposed rule Gl endoscopy RVU [PDF file]. The Centers for Medicare and Medicaid Services; 2019: Available at: https://webfiles.gi.org/links/policy/2020\%20MPFS\%20Proposed\%20Rule\%20Gl\% 20endoscopy\%20RVUs_for\%20members.pdf

[9] Procedural and reimbursement guide gastroenterology [PDF file]. Boston Scientific. American Medical Association; 2019: Available at: https://www.bostonscientific.com/content/dam/bostonscientific/ Reimbursement/Gastroenterology/2019/2019-GI-Procedural-Reimbursement-Guide.pdf

[10] Medtronic reimbursement support program [PDF file]. Medtronic and Center for Medicare and Medicaid Services; 2020: Available at: https://www.medtronic.com/content/dam/covidien/library/us/en/ services-support/reimbursement-guide-gih-solutions.pdf

[11] CMS releases payment information for endoscopic fundoplication for GERD. 03.11.2015 Available at: https://www.healio.com/news/gastroenterology/20151103/cms-releases-payment-information-forendoscopic-fundoplication-for-gerd

[12] 2020 Medicare reimbursement rules: impact to Gl practice. American Gastroenterological Association. American Gastroenterological Association and Center for Medicare and Medicaid Services; 2020: Available at: https://gastro.org/news/2020-medicare-reimbursementrules-impact-to-gi-practice/ 
[13] Belle A, Barret M, Bernardini D et al. Impact of the COVID-19 pandemic on the gastrointestinal endoscopic activity in France. Endoscopy 2020; 52: $1111-1115$
[14] Capurso G, Archibugi L, Vanella G et al. Infection control practices and outcomes of endoscopy units in the lombardy region of Italy: a survey from the Italian Society of Digestive Endoscopy during COVID-19 spread. J Clin Gastroenterol 2020: doi:10.1097/MCG.0000000000 001440 